Advanced Studies in Theoretical Physics

Vol. 12, 2018, no. 6, 257 - 265

HIKARI Ltd, www.m-hikari.com

https://doi.org/10.12988/astp.2018.8625

\title{
Conductive-Convective Heat Transfer in an Inclined Enclosure with Vertical Partition
}

\author{
Marhama Jelita \\ Department of Electrical Engineering \\ Faculty of Science Technology \\ State Islamic University of Sultan Syarif Kasim \\ 28293 Pekanbaru, Riau, Indonesia \\ Rika Taslim \\ Department of Industrial Engineering \\ Faculty of Science Technology \\ State Islamic University of Sultan Syarif Kasim \\ 28293 Pekanbaru, Riau, Indonesia
}

Copyright (C) 2018 Marhama Jelita and Rika Taslim. This article is distributed under the Creative Commons Attribution License, which permits unrestricted use, distribution, and reproduction in any medium, provided the original work is properly cited.

\begin{abstract}
Conductive-convective heat transfer in a square enclosure are studied numerically in the present article. The hot and cold walls are making an angle with horizontal direction. The Galerkin weighted residual finite element method has been used to solve the governing partial differential equations. In numerical simulations, the partition thickness, the orientation angle and the Rayleigh number are considered. It is found that the flow circulation decreases with decreasing the orientation angle and the maximum heat transfer rate is obtained near $\varphi=60^{\circ}$ for the considered partition thickness.
\end{abstract}

Keywords: conjugate convection, finite element method, comsol 


\section{Introduction}

Thermally driven flow and heat transfer in a differentially heated enclosures from side or below has received considerable attention over the past few decades, largely due to a wide variety of applications, which include building technology, electronic boxes, solar collector technology, energy storage, nuclear reactor technology, etc. Comprehensive theoretically or experimentally studies have been conducted by many authors. The topic of these studies is mostly enclosure bounded or partitioned by solid walls with zero thickness. In some situations, the conductivities of the enclosure walls and the fluid inside are comparable and the wall thickness is finite, for example in the high performance insulation for buildings. This coupled conduction-convection problem is called as conjugate convection. [1] studied a rectangular enclosure with a vertical partition and filled with air. They found that partitioning is an effective method of reducing heat transfer and the maximum reduction in heat transfer occurs when the partition was placed midway between the vertical walls. The heat transfer rate was found considerably attenuated in a partitioned enclosure in comparing with that for non-partitioned enclosure as reported by [2]. [3] studied numerically and experimentally enclosures with multiple vertical partitions and showed that the Nusselt number is inversely proportional to the number of partitions. [4] investigated the conductive multiple partitions and side walls and found the mean Nusselt number decreases with increasing of partition number. [5] compared the numerical and experimental results for a cubic enclosure without and with a partition. They concluded that the introduction of a complete vertical partition reduces convective heat transfer from $59.1 \%$ to $63.6 \%$ in the range of Rayleigh numbers $38,000<R a<369,000$. [6] reported the average Nusselt number increases with decreasing of thermal resistance of the partition and the partition thickness has little effect on heat transfer. [7] studied a vertically divided square enclosure by a solid partition into air and water regions and found that filling of fluid into chests is important for obtaining maximum heat transfer and energy saving. [8] addressed on the optimization of heat transfer rate by varying the partitions location, partitions

size and thermal conductivity ratio. Recently, [9] concluded that the partition position has a negligible effect on the average Nusselt number.

To the best of our knowledge, no report has been obtained for conductiveconvective heat transfer in an inclined square enclosures partitioned by conductive wall. Controlling the heat transfer using a partition and varying the orientation angle are essential in industrial applications or in a building construction. The aim of this work is to solve numerically the conductive-convective heat transfer in an enclosure with vertical partition. The hot and cold walls are making an angle with horizontal direction. 


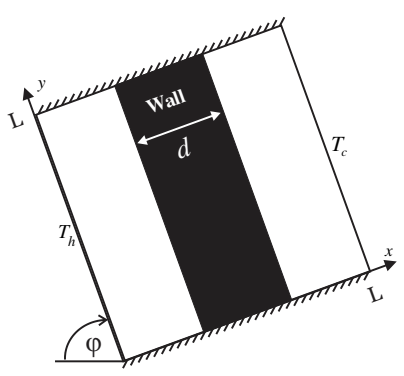

Figure 1: Schematic representation of the model.

\section{Mathematical Formulation and Solution}

A schematic diagram of an inclined square enclosures with sides of length $L$ is shown in Figure 1. The hot wall is making an angle of $\varphi$ with horizontal direction. For $\varphi=0^{\circ}$, hot wall becomes the bottom wall whereas for $\varphi=$ $90^{\circ}$, it becomes the left vertical wall. Opposite to the hot wall is the cold wall. Other two walls are maintained at adiabatic condition. Thermophysical properties of the fluid in the flow field are assumed to be constant except the density variations causing a body force term in the momentum equation. The Boussinesq approximation is invoked for the fluid properties to relate density changes to temperature changes, and to couple in this way the temperature field to the flow field. Under the above assumptions, the governing equations for steady natural convection flow using conservation of mass, momentum and energy can be written as

$$
\frac{\partial u}{\partial x}+\frac{\partial v}{\partial y}=0
$$




$$
\begin{gathered}
u \frac{\partial u}{\partial x}+v \frac{\partial u}{\partial y}=-\frac{1}{\rho} \frac{\partial p}{\partial x}+\nu\left(\frac{\partial^{2} u}{\partial x^{2}}+\frac{\partial^{2} u}{\partial y^{2}}\right) \\
-g \beta\left(T_{f}-T_{c}\right) \cos \varphi \\
u \frac{\partial v}{\partial x}+v \frac{\partial v}{\partial y}=-\frac{1}{\rho} \frac{\partial p}{\partial y}+\nu\left(\frac{\partial^{2} v}{\partial x^{2}}+\frac{\partial^{2} v}{\partial y^{2}}\right) \\
+g \beta\left(T_{f}-T_{c}\right) \sin \varphi \\
u \frac{\partial T_{f}}{\partial x}+v \frac{\partial T_{f}}{\partial y}=\alpha\left(\frac{\partial^{2} T_{f}}{\partial x^{2}}+\frac{\partial^{2} T_{f}}{\partial y^{2}}\right)
\end{gathered}
$$

and the energy equation for the solid divider are:

$$
\frac{\partial^{2} T_{w}}{\partial x^{2}}+\frac{\partial^{2} T_{w}}{\partial y^{2}}=0
$$

where the subscripts $f$ and $w$ stand for the fluid and the wall respectively. No-slip condition is assumed at all the solid-fluid interfaces. The values of the velocity are zero in the solid regions and on the solid-fluid interfaces. Continuity of the heat flux at the solid-fluid interfaces in both enclosures

$$
\frac{\partial T_{f}}{\partial y}=K_{r} \frac{\partial T_{w}}{\partial y}
$$

where $K_{r}=k_{w} / k_{f}$ is the thermal conductivity ratio. At the same time, continuity of the temperature at the solid-fluid interfaces is represented by $T_{f}=T_{w}$.

The governing equations with the boundary conditions are solved numerically by employing Galerkin weighted residual finite element method. The basic idea of this method is dividing the whole domain into smaller elements of finite dimensions called finite elements. The solution domain is generated into finite element meshes, which are composed of non-uniform triangular elements. The triangular mesh distribution calibrates for fluid dynamics condition. We consider the Laminar Flow (spf) for the continuity and momentum equation and Heat Transfer (Ht) for the convection and conduction equations. Figure 2 shows the snapshot of the Comsol program illustrating the triangular mesh distribution with an extra fine element size and the model builder settings.

After the convergence is reach then the Nusselt number or the heat transfer are calculated. We present the flow circulation using the streamfunction which is derived from the first derivative of velocities.

\section{Results and Discussion}

The analysis in the undergoing numerical investigation are performed in the following range of the associated dimensionless groups: the partition thickness, $0.0 \leq D \leq 1.0$, the inclination angle of the enclosure, $0^{\circ} \leq \varphi \leq 90^{\circ}$ and the 


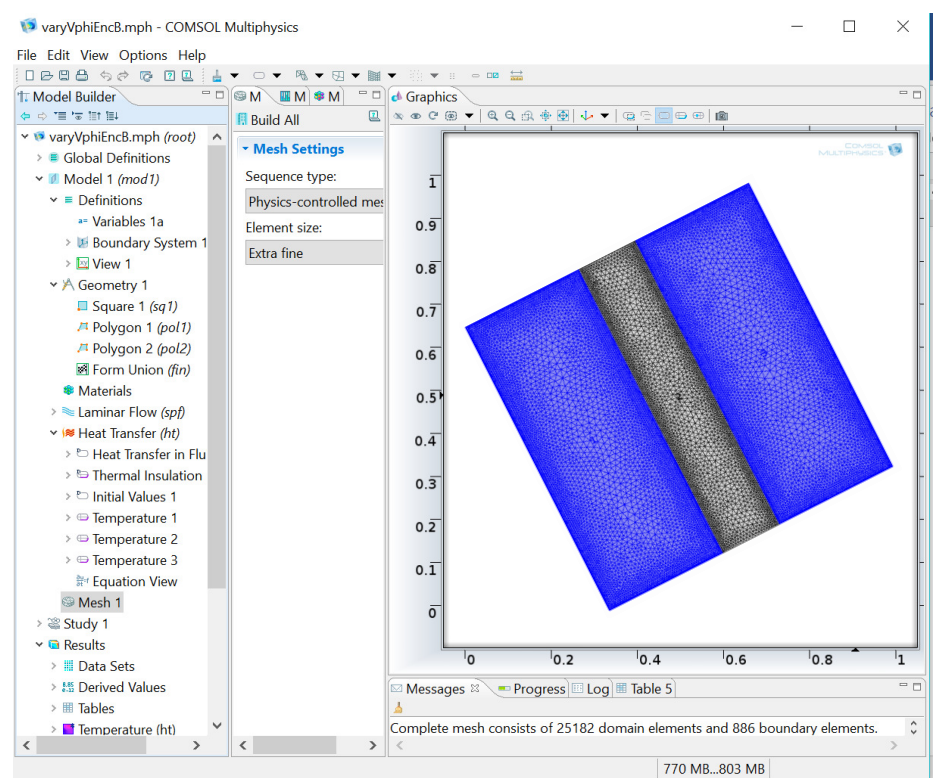

Figure 2: Snapshot of the Comsol program. 
Rayleigh number $10^{3} \leq R a \leq 10^{6}$. The Prandtl number is fixed at $\operatorname{Pr}=$ 0.71 and $K_{r}=1$. The flow and temperature fields are presented in terms of streamline and isotherms contours, respectively. The heat transfer rate is represented by the average Nusselt number.

Figure 3 shows the streamlines and isotherms to show the effects of inclination angle on flow fields and thermal distribution for $D=0.2$. Some of heat released by the hot surface and travels with the fluid in the hot portion, the partition wall released the heat again to fluid in the cold portion and it terminates at the cold surface. These create rotating cells circulation. Flow strength measured by the level labels. The flow circulation decreases with decreasing the orientation angle. The monocellular flow in the hot portion are negative dilation of the monocellular flow in the cold portion. The core vortex of the hot partition occurs at lower part while the core vortex of the cold partition occurs at upper part. For all angles, the isotherms in the solid partition are almost parallel with hot and cold surfaces. This is an evident that the partition acts as a thermal barrier.

Variations of the average Nusselt number is shown Fig. 4 for different values of $R a$ with constant values of $D=0.2$. Obviously, the average Nusselt number is increased as the Rayleigh number increases for any orientation angle. The average Nusselt number profile exhibit parabolic curve for the both cases except at the $R a=10^{3}$. This due to at $R a=10^{3}$, the both morphology stay in the conduction heat transfer mode at any orientation angle. At $R a=10^{4}$, The average Nusselt number remains 1, indicating almost no convection occur inside the enclosure. For higher buoyancy force, the partition wall blocks the convective flow at $\varphi=0^{\circ}$ or Bénard convection problem. Later, the partition conducts heat intensively from the hot fluid at higher gravitational force or higher temperature difference. The maximum heat transfer rate is obtained near $\varphi=60^{\circ}$.

\section{Conclusions}

In the present numerical simulations, we have studied the partitioned enclosure with different orientation. The governing equations are solved numerically using the built-in finite element method of COMSOL. Detailed computational results for flow and temperature fields and the heat transfer rate have been presented in the graphical forms. The inclination angle and Rayleigh number affected the shape of streamlines cells, isotherms lines and Nusselt number profiles. We found that the flow circulation decreases with decreasing the orientation angle and the maximum heat transfer rate is obtained near $\varphi=60^{\circ}$. 


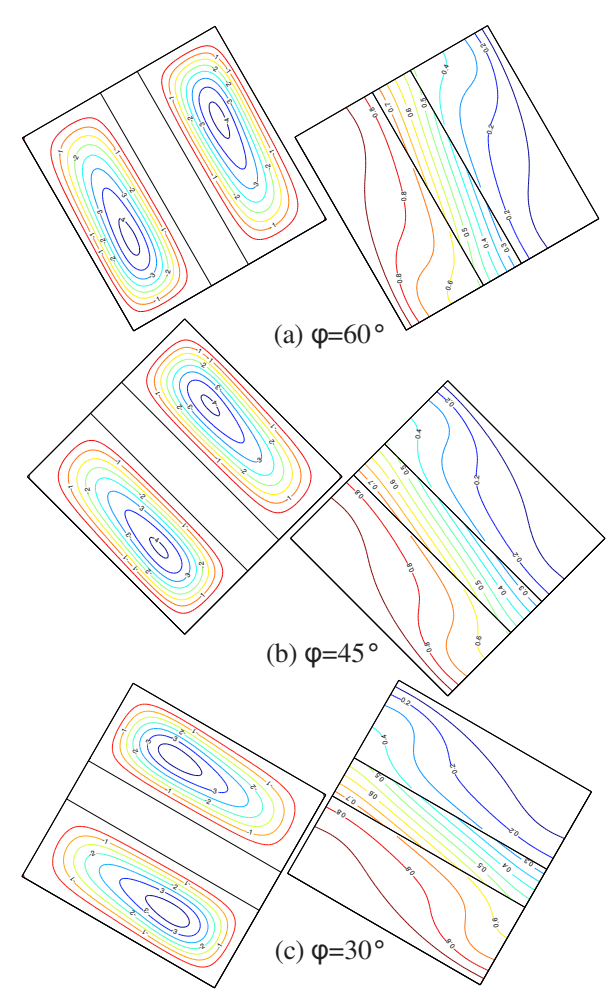

Figure 3: Streamlines (left) and isotherms (right) evolutions for several inclination angles at $D=0.2$ and $R a=10^{5}$. 


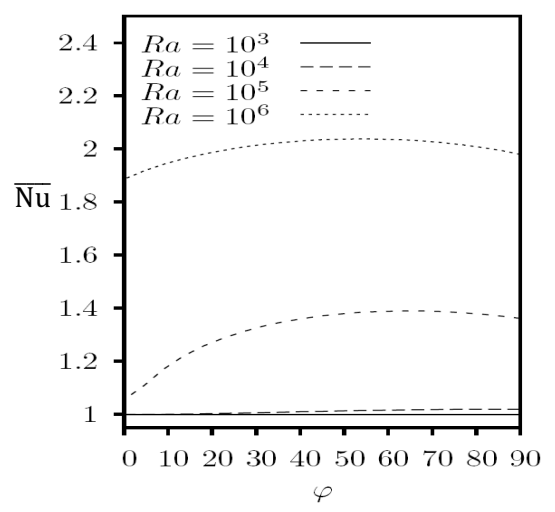

Figure 4: Variation of average Nusselt number with $\varphi$ for different $R a$ at $D=0.2$. 


\section{References}

[1] T.W. Tong and F.M. Gerner, Natural convection in partitioned air-filled rectangular enclosures, Int. Comm. Heat Mass Transf., 13 (1986), 99-108. https://doi.org/10.1016/0735-1933(86)90076-x

[2] C.J. Ho and Y.L. Yih , Conjugate natural heat transfer in an air-filled rectangular cavity, Int. Comm. Heat Mass Transf., 14 (1987), 91-100. https://doi.org/10.1016/0735-1933(87)90011-x

[3] T. Nishimura, M. Shiraishi, F. Nagasawa and Y. Kawamura, Natural convection heat transfer in enclosures with multiple vertical partitions, Int. J. Heat Mass Transf., 31 (1988), 1679-1686.

https://doi.org/10.1016/0017-9310(88)90280-3

[4] H. Turkoglu and N. Yucel, Natural convection heat transfer in enclosures with conducting multiple partitions and side walls, Heat Mass Transf., 32 (1996), 1-8. https://doi.org/10.1007/s002310050084

[5] D.M.C. Dzodzo, M.B. Dzodzo and M.D. Pavlovic, Laminar natural convection in a fully partitioned enclosure containing fluid with nonlinear thermophysical properties, Int. J. Heat Mass Transf., 20 (1999), 614-623.

https://doi.org/10.1016/s0142-727x(99)00053-3

[6] K. Kahveci, A differential quadrature solution of natural convection in an enclosure with a partial partition, Numer. Heat Transf. Part A, 52 (2007), 1009-1026. https://doi.org/10.1080/10407780701364536

[7] H. F. Oztop, Y. Varol and A. Koca, Natural convection in a vertically divided square enclosure by a solid partition into air and water regions, Int. J. Heat Mass Transf., 52 (2009), 5909-5921.

https://doi.org/10.1016/j.ijheatmasstransfer.2009.07.016

[8] D.D. Zhang, J.H. Zhang, D. Liu, F.Y. Zhao and H.Q. Wang, Conjugate thermal transport enhancement for an air filled enclosure with heat conducting partitions using inverse convection methodology, Int. J. Heat Mass Transf., 102 (2016), 788-800.

https://doi.org/10.1016/j.ijheatmasstransfer.2016.06.030

[9] Mehdi Khatamifar, Wenxian Lin, S.W. Armfield, David Holmes and M.P. Kirkpatrick, Conjugate natural convection heat transfer in a partitioned differentially-heated square cavity, Int. Comm. Heat Mass Transf., 81 (2017), 92-103. https://doi.org/10.1016/j.icheatmasstransfer.2016.12.003

\section{Received: June 21, 2018; Published: September 12, 2018}

